\title{
Les tumeurs des organes génitaux externes de l'homme
}

\section{Tumours of the Male External Genitalia}

\author{
O. Bouchot $\cdot$ S. Culine \\ (C) Springer-Verlag France 2014
}

Pour quelles raisons consacrer un numéro thématique aux tumeurs des organes génitaux externes de l'homme ?

- Parce qu'il s'agit de tumeurs rares, pour lesquelles il est toujours utile d'avoir sous la main un document de synthèse ;

- parce que la gestion diagnostique et thérapeutique de ces tumeurs doit obéir à des règles de prise en charge très précises, pour lesquelles une coopération optimale entre urologues et oncologues est nécessaire ;

- parce que les taux de guérison sont importants, sous réserve d'une rigueur dans la démarche diagnostique et thérapeutique ;

- et parce que enfin les enquêtes de pratique ne cessent de nous démontrer que seulement un tiers des patients sont effectivement pris en charge dans les règles de l'art! Un triste constat qui souligne la nécessité d'une diffusion régulière des connaissances et d'une prise en charge dans des centres spécialisés.

Nous espérons que vous trouverez de l'intérêt à la lecture des chapitres de ce numéro, rédigés par des experts dans le domaine. Bonne lecture!

O. Bouchot $(\bowtie)$

Clinique urologique, CHU de Nantes,

place Ricordeau, BP 1005,

F-44093 Nantes cedex,

France

e-mail : obouchot@chu-nantes.fr

S. Culine $(\bowtie)$

Service d'oncologie médicale,

AP-HP, hôpital Saint-Louis,

université Paris-VII, Paris,

France

e-mail : stephane.culine@sls.aphp.fr 